\title{
ELECTRON MICROSCOPY OBSERVATIONS OF THE MORPHOLOGY OF PLANE-STRAIN DEFORMED COPPER*
}

J.W.H.G. Slakhorst and M.J. ten Bouwhuijs

Technical University Twente, Enschede, Holland.

(Received July 28, 1977)

\section{Introduction}

Since the introduction of electron microscopy in the field of texture research, a large amount of data has been gathered with respect to the structure and the orientation distribution in heavily rolled copper. Extensive orientation determinations of individual grains were carried out $(1,2)$ in order to clarify the nature of the deformation texture. Separate studies of the structure of the deformed matrix were made in order to determine possible nucleation mechanisms during recrystallization $(3,4)$. Until now less attention has been paid to the morphology of the deformed structure: the deformed structure in relation to its orientation (5).

The results presented here are part of a current research program with an emphasis on this combination. A plane-strain deformation mode was chosen in order to guarantee a better controlled deformation process, which produces textures more or less identical to the rolling texture.

The principal experimental results can be summarized as follows:

a) No transition bands, as observed by Walter and Koch (6) and $\mathrm{Hu}$ (3) and predicted by Dillamore et al. (7) seem to be created even in those orientation arrangements which seem particularly fit for their creation.

b) Deformation twinning apparentiy does not play a relevant role during the deformation process, as was predicted by wassermann (8).

c) Extended regions of the deformed structure are characterized by nearly common <110>-axes situated in the vicinity of the transversal plane, as was proposed by Verbraak $(9,10,11)$ on the basis of an analys is of the textures of rolled single crystals and polycrystalline copper.

\section{Experimental}

The polycrystalline copper used had a purity of $99,99 \%$. Initially, the as cast cylindrical specimen was subsequently deformed to a rectangular shape of $50 \times 35 \times 10 \mathrm{~mm}$ by pressing and rolling in various planes and directions, with intermediate heat treatments. A near random texture resulted. Plane-strain deformation was now applied in steps of $30,50,66,80$ and $91 \%$ (although Molykote was used as a lubricant, friction caused a not completely plane-strain deformation process). After each deformation step $\{200\}-,\{111\}-$ and $\{220\}-$ back-reflection pole figures were measured with a Philips texturegoniometer (pitch 50), and specimens for transmission electron microscopy of the planes perpendicuiar to the T.D., the C.P.N. (beyond $50 \%$ reduction) and the F.D.** (only at $91 \%$ reduction) were prepared by cutting small sections with a Buehler diamond cutting wheel and subsequent electrochemical thinning in a Struers Tenupol with D2-electrolyte. From $66 \%$ reduction, the remaining material was stored in liquid $\mathrm{N}_{2}$ to avoid recrystallization at ambient temperature (12).

* This work is part of the research program of the research group FOM-TNO of the "Stichting voor fundamenteel onderzoek der materie" (Foundation for fundamental research of matter-F.0.M.) and was made possible by financial supsupport from the "Nederlandse organisatie voor zuiver wetenschappelijk onderzoek" (Dutch organisation for pure research-Z.W.0.).

* T.D. is transversal direction; F.D. is free direction (to be compared with the roliing direction) and C.P.N. is the compressionplane normal (compare rolling plane normal). 


\section{Results}

Fig. 1 gives an example of the development of the texture. It can be concluded

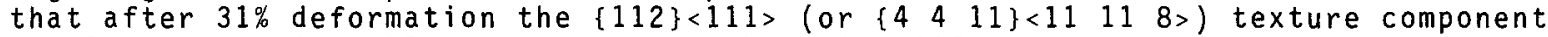
is already considerably stronger than the $\{110\}<112>$ component. This is in contradiction with the observations presented by Bunge et al. (13) and by Kallend and Davies (14), who observed a stronger $\{110\}<112>$ component up to $70 \%$ and $95 \%$ rolling deformation, respectively. The orientations measured with the T.E.M. were plotted in $\{200\}$-pole figures for comparison with the pole figures, obtained by $X-$ ray measurements (fig. 2 ).

The correlation between both methods is better at higher reductions. This can partly be explained from the fact that three different sections of the specimen were transmitted only after $91 \%$ deformation (resulting in 305 measured orientations). Furthermore a larger area has to be transmitted after low amounts of deformation, because of the fact that the areas with a uniform orientation distribution will be more extended. In these observations no Kikuchilines were observed, therefore an uncertainty of approximately 60 has to be allowed for the orientations plotted in fig. 2. In general, it can be said that the T.E.M.-observations bear great resemblance to similar results obtained by Gotthardt et al. (5): areas with dissimilar orientations are lying next to each other without any transition. After small amounts of deformation no transitionbands were detected, contrary to observations of Walter and Koch (6), who found transitionbands after small amounts of deformation gradually decreasing in thickness with increasing deformation. Heye and Wassermann (15) describe the splitting up of the $\{110\}<001>$ orientation into the symmetrical (110)|112| and (110)|112|orientations. A transitionband should be expected between these deformation bands.
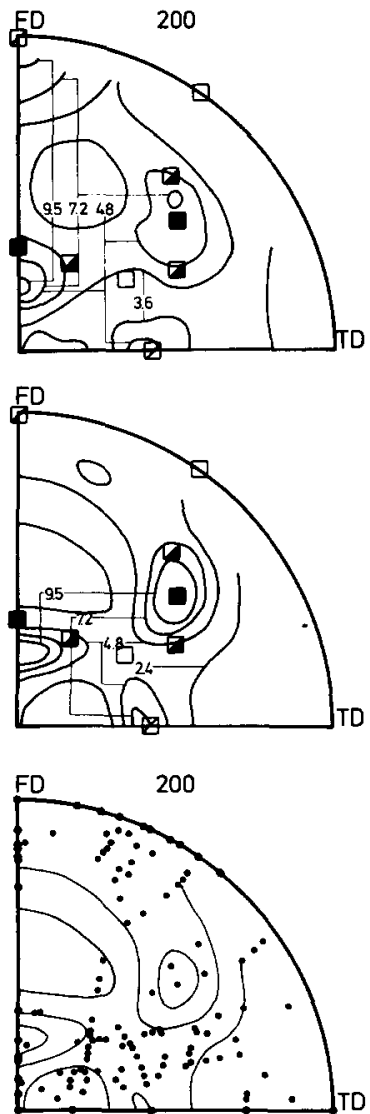
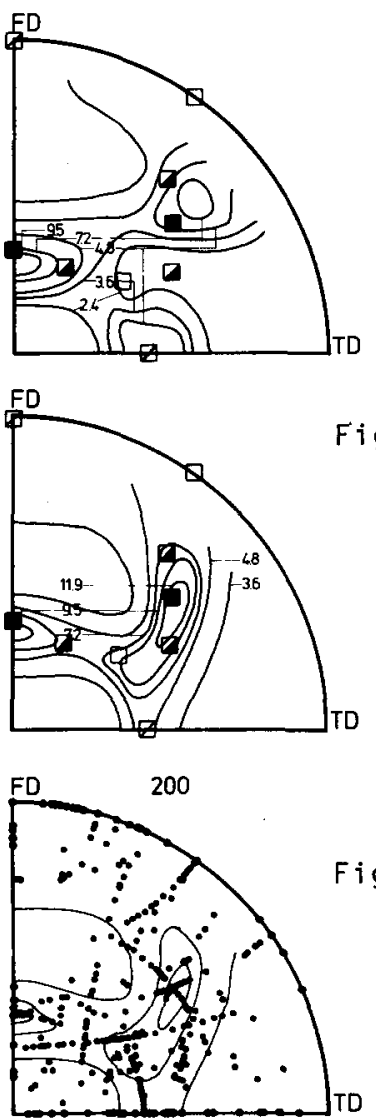

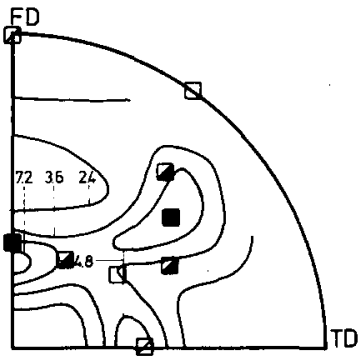

Fig. 1. Representative quarters of the $\{200\}$-pole figures of plane-strain deformed $\mathrm{Cu}$ after $30,50,66,80$ and $91 \%$ deformation.
Fig. 2. $\{200\}-p o l e$ plots comparing the E.M. and texture measurements after 80 and $91 \%$ deformation of Cu. 
Fig. 3

$\{110\}<112>$ orientation arrangement at $80 \%$ deformation.

$$
\begin{aligned}
& A=(110)[1 \overline{1} \overline{2}] \\
& B=(110)[2 \overline{2} \overline{5}] \\
& C=(110)[3 \overline{3} \overline{5}] \\
& 1=(110)[\overline{1} 1 \overline{2}] \\
& 2=(110)[\overline{7} 7 \overline{1} \overline{6}] \\
& 3=(110)[\overline{2} 2 \overline{5}] \\
& 4=(110)[\overline{1} 1 \overline{3}] \\
& 5=(110)[\overline{1} 1 \overline{5}]
\end{aligned}
$$

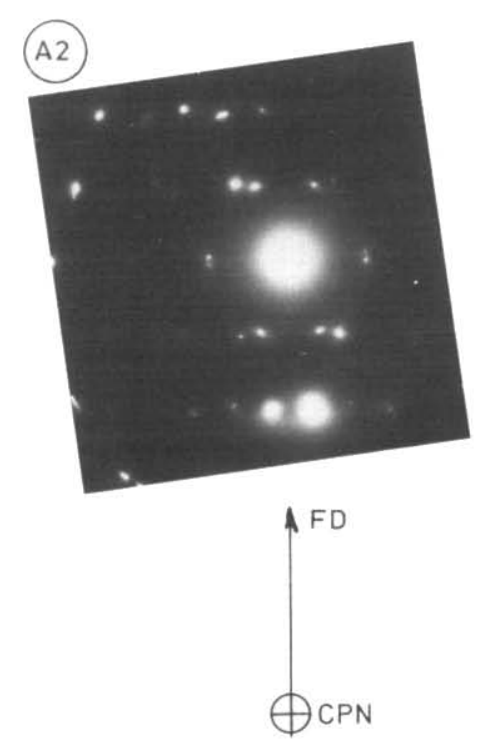

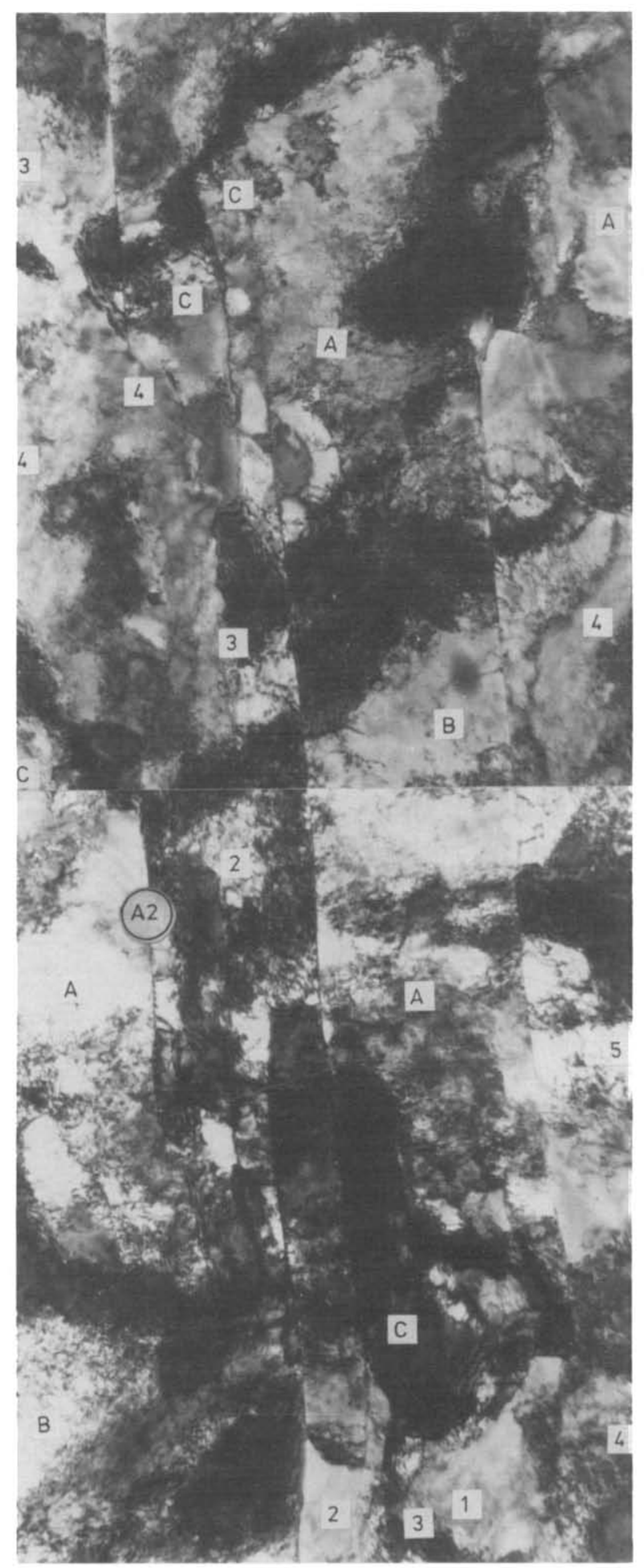




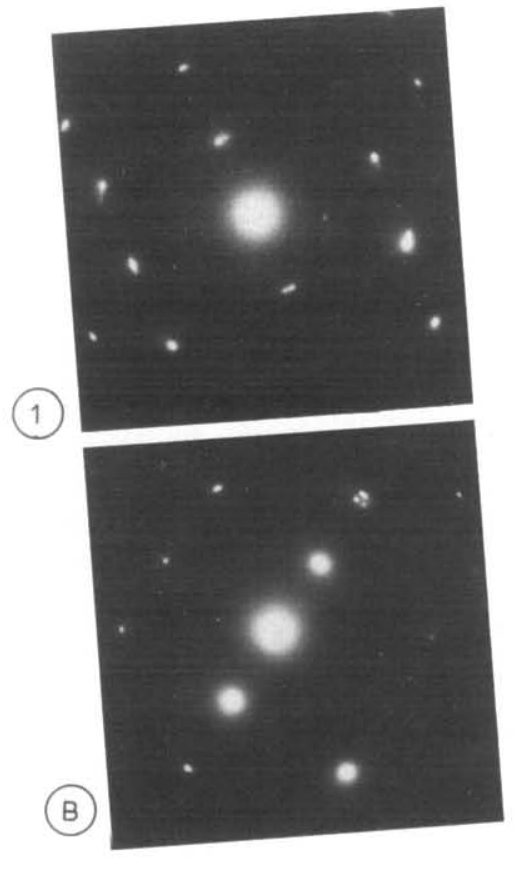

Fig. 4 :

Twin at $91 \%$ deformation; twinboundaries are indicated by arrows.

$$
\begin{aligned}
& A=\left(\begin{array}{lll}
4 & 14 \overline{9}
\end{array}\right)\left[\begin{array}{lll}
\overline{37} & 17 & 10
\end{array}\right] \\
& B=\left(\begin{array}{lll}
\overline{8} & \overline{8} & 5
\end{array}\right)\left[\begin{array}{lll}
\overline{7} & 3 & 2
\end{array}\right] \\
& 1=\left(\begin{array}{lll}
7 & 1 \overline{4}
\end{array}\right)\left[\begin{array}{lll}
\overline{1} & 3 & \overline{1}
\end{array}\right] \\
& 2=\left(\begin{array}{llll}
4 & 1 \overline{2}
\end{array}\right)\left[\begin{array}{lll}
1 & 2 & \overline{1}
\end{array}\right]
\end{aligned}
$$
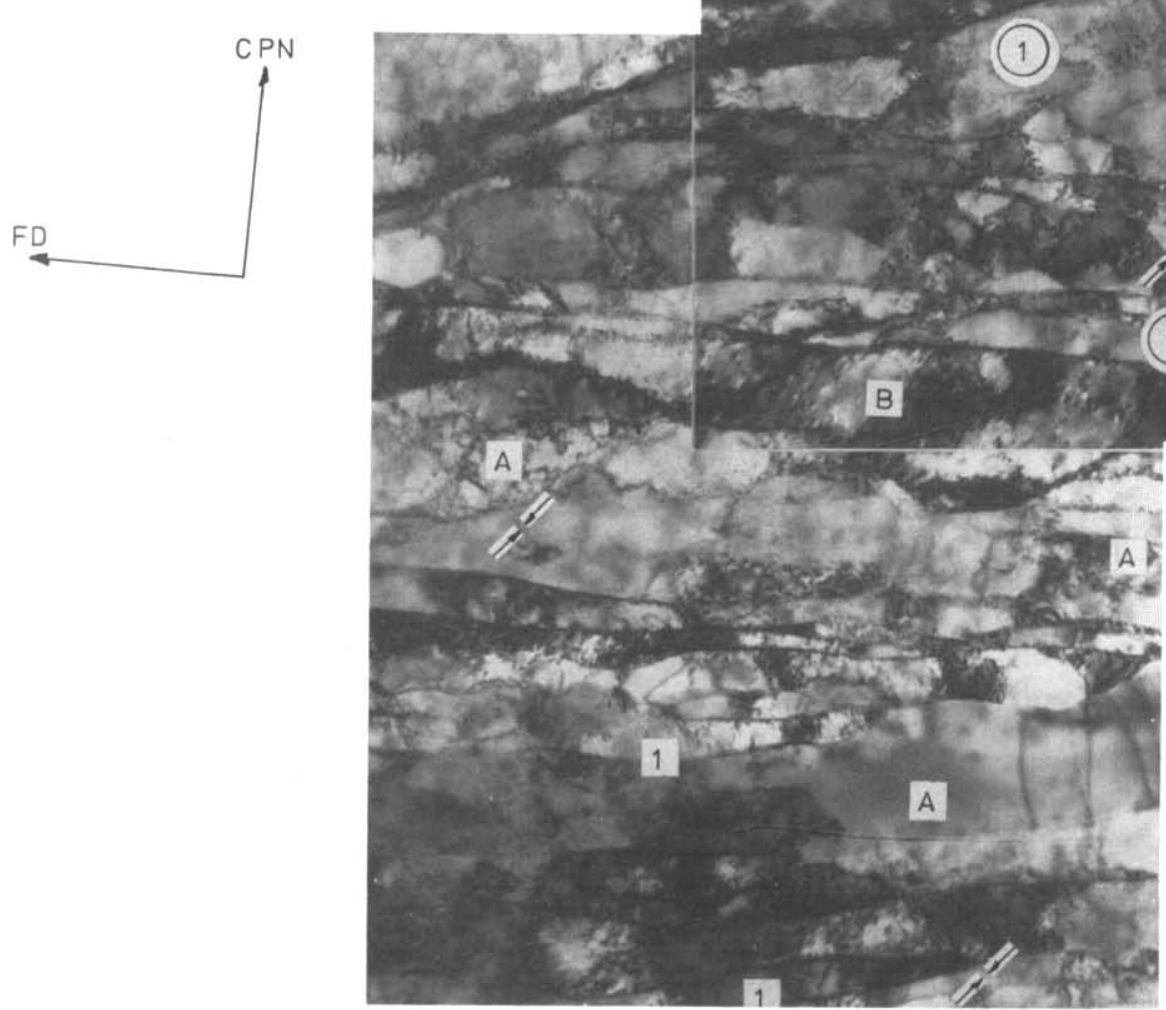


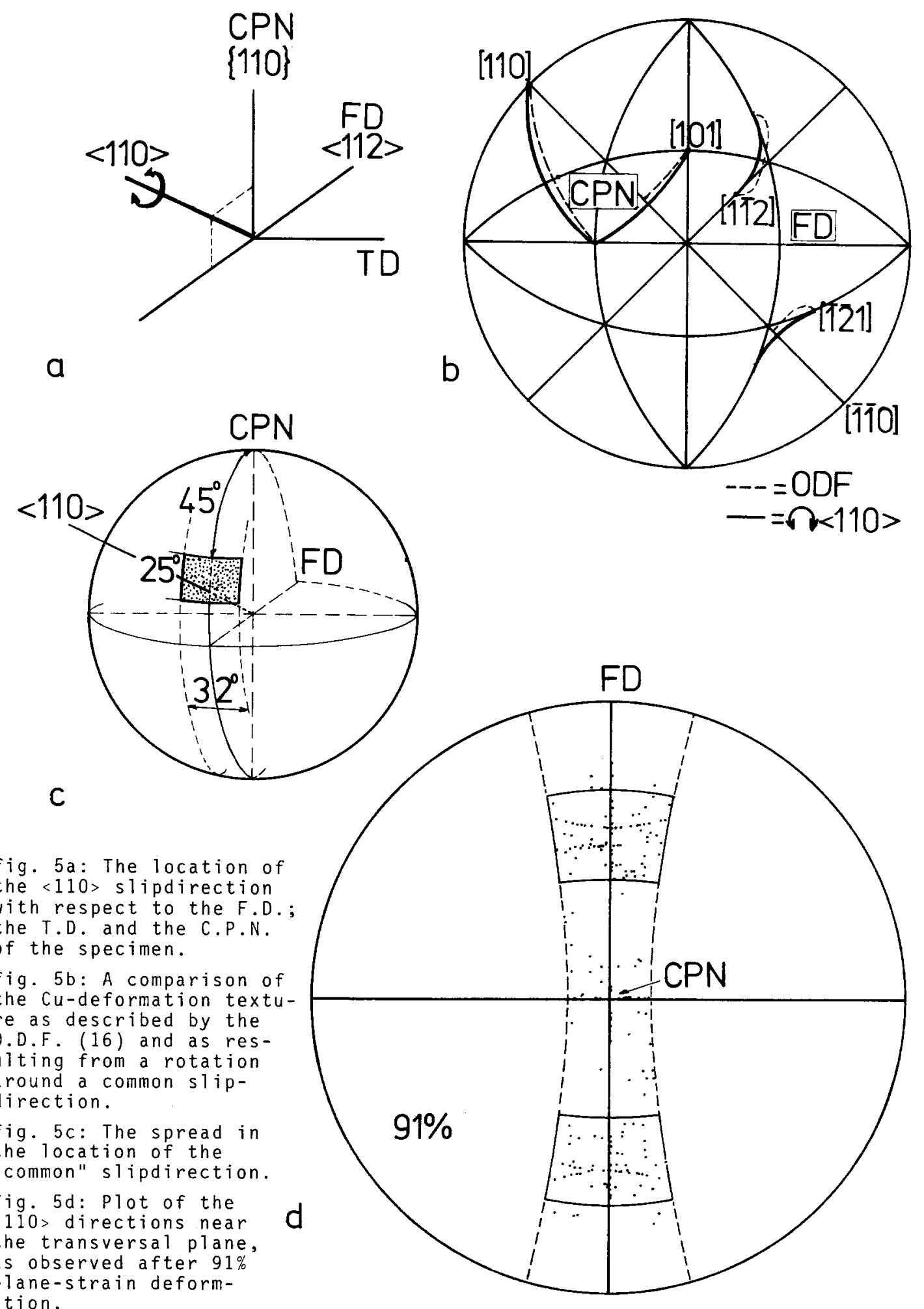

fig. 5a: The location of the <110> slipdirection with respect to the F.D.; the T.D. and the C.P.N. of the specimen.

fig. 5b: A comparison of the cu-deformation texture as described by the 0.D.F. (16) and as resulting from a rotation around a common slipdirection.

fig. 5c: The spread in the location of the "common" slipdirection.

fig. 5d: Plot of the $<110\rangle$ directions near the transversal plane, as observed after $91 \%$ plane-strain deformation. 
Fig. 3 shows a similar orientation arrangement after $80 \%$ deformation. It can be concluded from the matching diffraction pattern that the two distinct orientations are situated next to each other without a transitionband. Neither could this arrangement of orientations be created by mechanical twinning since the resolved shear stress on the twinning plane (the (III)-plane, i.e. the transversal plane) is zero.

On the whole, mechanical twinning was observed only occasionally. It should be born in mind however, that these twins are often difficult to detect, as can be seen from a representative example in fig. 4 .

None of the twins observed had the $\{552\}<115>$ orientation, as predicted by wassermann (8). This is, however, in good agreement with the results of verbraak and Slakhorst on Cu-single crystals (12).

Because of these observations mechanical twinning can hardly be expected to play a role during the development of the Cu-rolling texture. According to Verbraak $(9,10,11)$ the copper rolling texture should be described as a series of orientations obtained by the rotation from (110)|1I2| via (121)|1I1| to (011)|2I1| around their nearly common $|101|$ slip direction (i.e. the active slip direction which causes no broadening of the specimen), lying in the transversal plane (fig. 5a). It can be seen from a comparison with fig. $5 b$ that this description is in excellent agreement with the spread of orientations presented by the O.D.F. skeletonline (Bunge and Tobisch (16)).

For the purpose of a statistical evaluation a spread around the theoretical direction was chosen as shown in fig. 5 C. The T.E.M. observations on the $91 \%$ deformed specimens proved that $78 \%$ out of 305 orientations investigated had a $<110\rangle$ slip direction in this area of spread around the theoretical position (fig. 5d), whereas in a randomly oriented specimen this would hold for only $23 \%$ of the crystals. Furthermore, it should be noted that $11 \%$ of the orientations measured after $91 \%$ deformation was still between $\{110\}<001>$ and $\{110\}<112>$ and it can be expected that these orientations will complete their rotation to the stable $\{110\}<112>$ orientation at higher amounts of deformation. This will still further increase the percentage of crystals with a <110 direction in the area defined to about $90 \%$.

From these results it may be concluded that the macroscopic description of the cu rolling texture by Verbrak is also valid for a microscopic description of the arrangement of orientations in extended areas. It was suggested by verbraak $(10,11)$ that this orientational arrangement is of great significance for the nucleation of new orientations during recrystallization.

\section{References}

1. F. Haessner, U. Jakubowski and M. WiTkens, Mat. Sci. Eng., 1, 30 (1966).

2. K. Lücke, H. Perlwitz and W. Pitsch, Phys. Stat. Sol., 7,733 (1964).

3. H. Hu, Texture in research and practice, Proc. 1st Int. Conf. on Textures, Springer, Berlin (1969).

4. R.K. Ray, W.B. Hutchinson, and B.J. Duggan, Acta Met., 23, 831 (1975).

5. R. Gotthardt, G. Hoschek, 0. Reimold and F. Haessner, Texture, 1, 99 (1972).

6. J.L. Walter and E.F. Koch, Acta Met., 11,923 (1963).

7. I.L. Dillamore, P.L. Morris, C.J.E. Smith and W.B. Hutchinson, Proc. Roy. Soc., A. 329,405 (1972).

8. G. Wassermann, Z. Metal1k., 54, 61 (1963).

9. C.A. Verbraak, Thesis, Delft (1959).

10. C.A. Verbraak, Acta Met., 6, 580 (1958).

11. C.A. Verbraak, Z. Metalik., 51, 646 (1960).

12. C.A. Verbraak and J.W.H.G. Slakhorst, Scripta Met., 8, 217 (1974).

13. H.J. Bunge, J. Tobisch and W. Sonntag, J. Appl. Cryst., 4, 303 (1971).

14. J.S. Kallend and G.J. Davies, Texture, 1,51 (1972).

15. W. Heye and G. Wassermann, Z. Metallk., 59,617 (1968).

16. H.J. Bunge and J. Tobisch, Z. Metallk.,59, 471 (1968). 